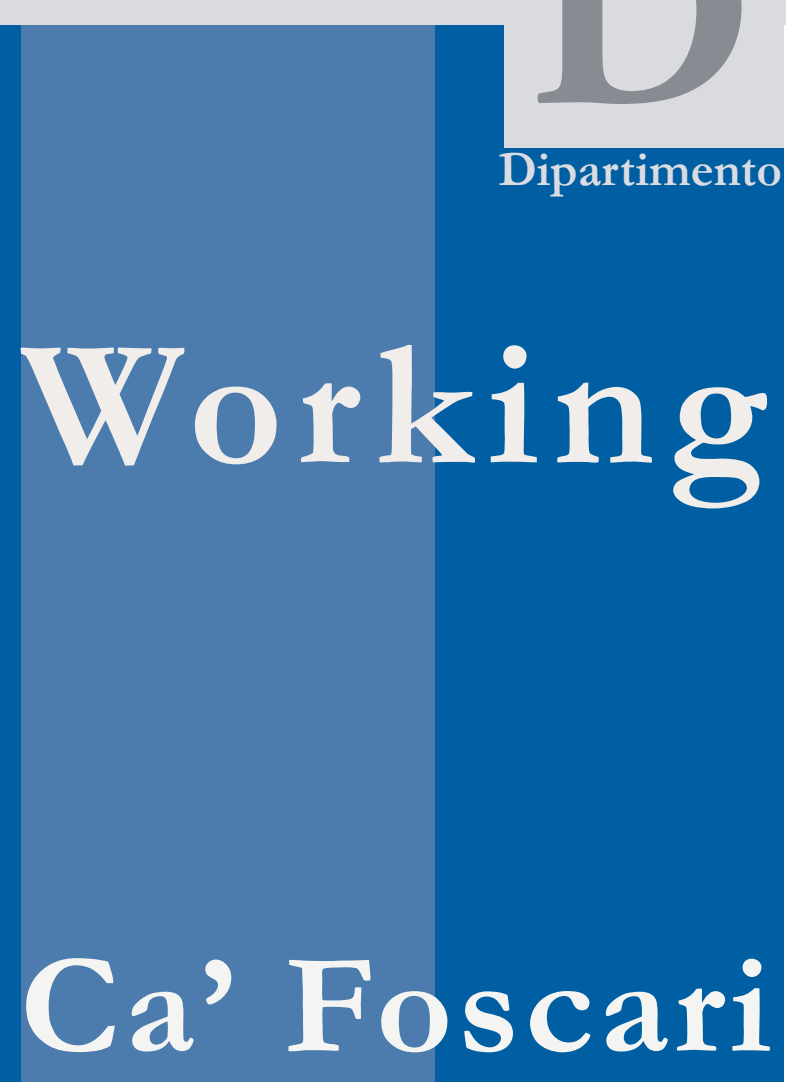

Paper

Department

of Economics

University of

Venice

Barbara Buchner

Carlo Carraro

Parallel Climate Blocs Incentives to cooperation in international climate negotiations 


\title{
Parallel Climate Blocs \\ Incentives to cooperation in international climate negotiations
}

\author{
Barbara Buchner \\ Fondazione Eni Enrico Mattei \\ Carlo Carraro \\ University of Venice, Fondazione Eni Enrico Mattei, CEPR and CEPS
}

First Draft: June 2005. Revised: July 2006

\begin{abstract}
There are increasing signals that countries that negotiate on GHG emission control are unlikely to sign and ratify a single climate protocol, even though almost all countries have subscribed the UNFCCC convention that sets the framework of international climate cooperation. In addition to the US decision not to ratify the Kyoto Protocol, New Zealand and Australia recently led to the formation of a new alliance in which technological cooperation is the main tool to achieve GHG emission control. In the U.S., some States on the Eastern coast are negotiating to adopt emission reduction targets and to establish a permit market despite the opposition of the federal government. Cooperation on climate policy is also the objective of recent negotiations between ASEAN countries. Given this background, this paper aims at examining whether the aforementioned events are simply the noise of a political process leading to a global agreement on climate change control or are instead consistent with some basic economic incentives that are pushing countries towards the formation of two (or more) parallel climate blocs. To this aim, this paper uses a well known integrated assessment climate-economy model to evaluate the incentives to cooperation in climate negotiations for the main world countries. A game-theoretic framework is adopted to analyse a country's incentive to belong to a climate coalition. In our setting, a given country can either join one of the existing climate coalitions or can propose a new one or can decide to free-ride on the other countries' cooperative abatement effort. We then analyse the characteristics of the main possible outcomes and assess which outcomes are most likely to prevail in future negotiations, at least as far as economic incentives are concerned.
\end{abstract}

\section{Keywords}

Agreements, Climate, Incentives, Negotiations, Policy

\section{JEL Codes}

C72, H23, Q25, Q28

\author{
Address for correspondence: \\ Carlo Carraro \\ Department of Economics \\ Ca' Foscari University of Venice \\ Cannaregio 873, Fondamenta S.Giobbe \\ 30121 Venezia - Italy \\ Phone: (++39) 0412349166 \\ Fax: (++39) 0412349176 \\ e-mail:ccarraro@unive.it
}

This Working Paper is published under the auspices of the Department of Economics of the Ca' Foscari University of Venice. Opinions expressed herein are those of the authors and not those of the Department. The Working Paper series is designed to divulge preliminary or incomplete work, circulated to favour discussion and comments. Citation of this paper should consider its provisional character.

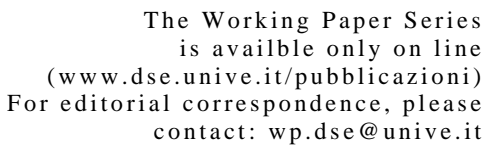

The Working Paper Series

is availble only on line (www.dse.unive.it/pubblicazioni)

For editorial correspondence, please

contact:wp.dse@unive.it

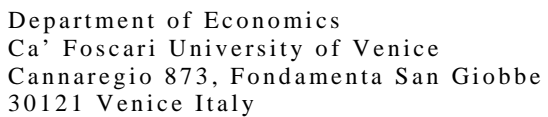

Department of Economics

Ca' Foscari University of Venice

Cannaregio 873, Fondamenta San Giobbe

30121 Venice Italy

Fax: ++390412349210 


\section{Parallel Climate Blocs* * INCENTIVES TO COOPERATION IN INTERNATIONAL CLIMATE NEGOTIATIONS}

\section{Introduction}

The lack of a credible strategy to include some large greenhouse gas emitters, in particular the US, China and India, among the signatories of the Kyoto Protocol has questioned the current approach to strive for a global top-down climate change protocol. Yet, countries across the world have committed to the overall idea behind climate policy by signing the United Nations Framework Convention on Climate Change (UNFCCC), which sets the framework to work against the climate change threat. At the same time, awareness on the potential scope of climate change seems to grow across the world countries, as evidenced for example by the relevance this topic had at the G8 summit in Gleneagles in the Summer 2005. However, consensus on the approach to design and implement climate change control appears to be missing.

Recent developments in international climate policy stress the growing importance of taking the single countries' incentives and specificities into account. After the top-down process of Kyoto negotiations, where overall binding emissions targets have been assigned to the different countries, there is an increasing tendency to focus more on domestic and/or bi-lateral climate-friendly activities than on global emissions targets. Initiatives by small groups of countries can also be observed.

In July 2005, the Asia-Pacific Partnership on Clean Development and Climate has stressed technology cooperation as a key tool to contribute to climate policy. This agreement - signed by the US, Australia, Japan, China, India and South Korea - constitutes a voluntary, technology-based initiative to reduce greenhouse gas emissions without legally binding emissions targets. Its basic idea is to cooperate to develop new technologies and to deploy these technologies in developing countries. Other bilateral agreements on technology and climate change indicate the attractiveness of this strategy. For example, in September 2005, the European Union and China agreed to strengthen cooperation and dialogue on climate change and energy issues, with a special focus on clean coal technology (Cf. Buchner and Carraro, 2005a, for a description of the main technological agreements).

\footnotetext{
* This paper is part of the research work being carried out by the Climate Change Modelling and Policy Unit at Fondazione Eni Enrico Mattei and has been presented at the CESifo David Bradford Memorial Conference on "The Design of Climate Policy". The authors are grateful to Christian Egenhofer, Frank Convery, Johan Eyckmans, Henry Tulkens, two anonymous referees, and the participants at the FEEM-Stanford Conference on "Post 2012 Climate Policy: Architectures and Participation Scenarios” in Venice, 20-21 June 2005 for helpful suggestions and remarks. The usual disclaimer applies.
} 
The emergence of cooperation between small groups of countries can be noticed also by observing some recent developments in emission trading markets. In Europe, the Emissions Trading Scheme was officially launched in January 2005. The system cover around 15,000 installations in 25 countries and 6 major industrial sectors. Similarly, in August 2005, Canada announced the establishment of the Canadian Offset System. The Canadian government released its plan to encourage the creation of greenhouse gas emission reduction (GhG ER) domestic offsets, including the introduction of domestic emissions trading. At the end of September 2005, after months of internal government debates, also Japan’s Ministry of Environment announced its decision to set up a voluntary domestic emissions trading scheme, beginning in April 2006 with an initial coverage of 34 manufacturing companies.

At the same time, a wide variety of initiatives are in preparation at state and local level in the U.S., notwithstanding the opposition of the federal government to commit to binding international climate policy goals. In particular, several US states in the North East are attempting to set up an emissions trading scheme within the Regional Greenhouse Gas Initiative (RGGI), whereas numerous other states are implementing serious climate change strategies, as evidenced by California's call for a return to 1990 emissions levels by 2020. California is also the leader in the negotiations on a GHG trading scheme amongst three Pacific Coast States. In addition, New Mexico has become the first US state in September 2005 to sign up for voluntary emissions trading at the Chicago Climate Exchange, pledging to reduce its state government’s GHG emissions by 4 per cent by 2006.

The aforementioned policy developments signal the evolution of climate policy towards a set of multiple initiatives/agreements, in which a single country or a small group of countries unilaterally adopt policy measures to combat climate change. Yet, to our knowledge, no sound analysis has been performed in order to verify whether the events described above correspond to some basic underlying economic incentives that make it convenient for countries to commit to unilateral or small group policy measures or whether these tendencies simply reflect a new round of political episodes, i.e. the noise of a political process leading to a global agreement on climate change control.

Therefore, the main objective of this paper is to appropriately assess the main world countries' incentives to cooperate on GHG emission control, i.e. to participate in a climate coalition. This will enable us to understand whether the equilibrium of the policy process is actually characterised by a global climate coalition or rather by a set of fragmented climate regimes in which several small coalitions emerge. To this aim, this paper uses the FEEM-RICE model, a well known integrated assessment climate-economy model, and some tools of non-cooperative coalition theory to identify the equilibrium coalition structure that could emerge out of climate negotiations. In order to be able to unravel the basic economic incentives, we keep the policy framework as simple as possible, without 
capturing all the details of the recent policy developments. For example, in our setting, climate negotiations only centre around the stringency of the environmental target.

The main assumption in our analysis, and its major novel feature with respect to previous empirical analyses of climate policy, is that a global agreement is only one of the possible outcomes of climate negotiations. According to their own economic interests, countries are also free to form regional or sub-global agreements. In particular, a given country can either join one of the existing climate coalitions or can propose a new one or can simply decide to free-ride on the other countries' cooperative abatement efforts. Each climate coalition allows the implementation of the flexible mechanisms (emission trading) within the coalition in order to guarantee an efficient implementation of the environmental targets adopted within the coalition. This framework is meant to mimic the recent developments in climate policy described above, where we can observe the emergence of various carbon markets across the world, with countries participating in one of these markets (Cf. Victor, 2006 for a discussion of this policy framework).

Let us finally stress that the focus of our research is on economic incentives faced by countries. There are several other political, cultural, and environmental factors that could influence a country's decision to join a given climate coalition, which will not be addressed in this paper. However, the economic dimension of climate negotiations has evolved as one of the key aspects in the international climate debates (and has often been considered as the most important one in the US). As a consequence, this paper can provide a relevant, albeit partial, contribution to the analysis of the future evolution of international climate policy.

The structure of the paper is as follows. Section 2 will review the main results that coalition theory provides on the formation of multiple coalition in a non-cooperative framework. Section 3 will describe the theoretical setting and the empirical model used for our analysis. Section 4 will present our main results. Finally, a concluding section will present the main policy implications of our work and will discuss some future research directions.

\section{Regional and sub-global climate blocs. Lessons from coalition theory.}

The strategic choice of players who decide whether or not to form a coalition with other players and, if they do, with which specific players to cooperate, has been the subject of recent research in game theory. ${ }^{\dagger}$ Many of these recent studies are based on a non-cooperative approach where binding

\footnotetext{
† Most papers have been presented at the annual workshops of the Coalition Theory Network (see www.feem.it/ctn). Some of them are published in Carraro (2003) and in Demange et al. (2005).
} 
commitments are excluded. This approach is particularly suitable for analysing the likely outcomes of future negotiations on climate change control, because no supra-national authority exists that can force countries to adopt policy measures to reduce their GHG emissions. Let us therefore examine the indications that the non-cooperative theory of coalition formation provides for the analysis of climate negotiations.

The study of coalition formation poses three basic questions (Cf. Bloch 1996): (i) which coalitions will be formed ? (ii) how will the coalitional worth be divided among members ? (iii) how does the presence of other coalitions affect the incentives to cooperate? The traditional cooperative game theory (Cf. Aumann and Drèze, 1974) focuses on the second question - the division of the payoff between coalition members. The first question has been assumed away in most cooperative game theory and the third one is simply ignored, since the coalitional function cannot take into account externalities among coalitions.

These limitations have led to the emergence of a new strand of literature describing the formation of coalitions as a non-cooperative, voluntary, process. In the non-cooperative approach, a player's decision to join a coalition is often modelled as a two stage game. In the first stage, a player independently decides whether or not to join, by anticipating the consequence of his/her decision on the economic variables under control. In the second stage, he/she sets the value of these variables, given the coalition structure formed in the first stage. Under the simplifying assumption that the second stage equilibrium is unique for any coalition structure, the first stage game can be reduced to a partition function, which assigns a value to each coalition in a coalition structure as a function of the entire coalition structure. This enables us to capture the important effects of externalities across coalitions.

The theoretical literature on the non-cooperative coalition formation has shown that, even without any commitment to cooperation and even in the presence of positive spillovers (i.e. in the case in which the formation of a coalition by some players increases the payoff of the players outside the coalition, as for public good provision), countries may form a coalition.

The equilibrium coalition structure depends on several key assumptions, i.e. the membership rule, the order of moves, the players' conjectures, the slope of their reaction functions (Cf. Carraro and Marchiori, 2003). Nevertheless, some conclusions seem to be robust with respect to these assumptions and the related equilibrium concepts. For example, if a treaty is signed by many countries (i.e. a large coalition is formed) the amount of public good provided by the coalition (e.g. the amount of GHG abatement) is very close to the non-cooperative business-as-usual one (Barrett, 2002). As far as the goal of this paper is concerned, the most important conclusion is as follows. If countries are free to 
decide not only whether or not to sign a treaty but also which treaty (i.e. which coalition to join), there is generally more than one coalition at the equilibrium. For example, in the case of trade negotiations, there may be several trade blocs. In the case of environmental negotiations, several regional or subglobal climate agreements.

This conclusion can be found for example in Bloch $(1995,1996)$, Ray and Vohra $(1997,1999)$, Yi (1997, 2003) and Yi and Shin (1995). The models used in these studies analyse the formation of multiple coalitions by adopting different notions of stability. Bloch $(1995,1996)$ examines an infinitehorizon "coalition unanimity" game, in which a coalition forms if and only if all potential members agree to form the coalition. Ray and Vohra (1997) assume the "equilibrium binding agreement" rule, under which coalitions are allowed to break up into smaller sub-coalitions only. Yi and Shin (1995) investigate the "open membership" game, in which non-members can join an existing coalition even without the consensus of the existing members. Different membership rules lead to different predictions about stable coalition structures (Cf. Carraro and Marchiori, 2003). For example, the "open membership" rule is unlikely to support the grand coalition as an equilibrium outcome. The equilibrium coalition structure is generally very fragmented. By contrast, the "coalition unanimity" rule and the "equilibrium binding agreements" rule support more concentrated coalition structures at the equilibrium, but quite often not the grand coalition (Cf. Finus and Rundshagen, 2003).

The above results have been used by Carraro $(1998,1999)$ to argue that the Kyoto Protocol was unlikely to be signed by all the relevant countries and that the emergence of parallel climate blocs was likely. However, economists are not alone in suggesting that climate negotiations may lead to multiple fragmented climate agreements. Some indications that multiple climate blocs could be the outcome of future climate negotiations can also be found in the political science and legal literature (see, for example, Egenhofer, Hager and Legge, 2001; Stewart and Wiener, 2003; Reinstein, 2004). As Victor (2006) says: "evidence that governments are taking the climate challenge seriously will come in the form of fragmented and variegated markets rather than integrated systems”.

However, no economic analysis has already quantified the incentives for negotiating countries to form multiple climate coalitions and identified which countries belong to which coalition. This paper is a first attempt to fill this gap.

\section{The Model}

\subsection{The Theoretical Framework}

Coalition formation is modelled as a two-stage game. There are $n$ players $N=\{1, \ldots, n\}$ that are countries or world regions in our empirical model and which we simply refer to as countries in the 
following discussion. In the first stage, countries choose their membership: a country can either join coalition $S_{i}$ and become a signatory or remain a singleton and non-signatory. These decisions lead to a coalition structure $S=\left(S_{1}, S_{2}, \ldots S_{k}, l_{k}\right)$, if $k$ coalitions form and the remaining players are singleton.

In the second stage, countries choose their economic strategies. At this stage, it suffices to denote the vector of economic strategies by $\omega(S)=\left(\omega_{1}(S), \ldots, \omega_{n}(S)\right)$, given that $k$ coalitions have formed in the first stage; we can also note that in the second stage countries receive individual payoffs $\pi_{\mathrm{i}}(\omega(\mathrm{S}))$ that depend on the economic strategies of all countries. ${ }^{\ddagger}$

The subgame-perfect equilibria of this two-stage game can be computed by backward induction. To do this, it is sufficient for strategies to constitute a Nash equilibrium at every stage. For the second stage, this entails that economic strategies form a Nash equilibrium between coalitions $S_{i}, i=1, \ldots k$, and nonsignatories. ${ }^{\S}$ That is:

$$
\begin{aligned}
& \sum_{\mathrm{i} \in \mathrm{S}} \pi_{\mathrm{i}}\left(\omega_{\mathrm{S}}^{*}(\mathrm{~S}), \omega_{-\mathrm{S}}^{*}(\mathrm{~S})\right) \geq \sum_{\mathrm{i} \in \mathrm{S}} \pi_{\mathrm{i}}\left(\omega_{\mathrm{S}}(\mathrm{S}), \omega_{-\mathrm{S}}^{*}(\mathrm{~S})\right) \quad \forall \omega_{\mathrm{S}}(\mathrm{S}) \text { and } \\
& \forall \mathrm{i} \notin \mathrm{S}: \pi_{\mathrm{i}}\left(\omega_{\mathrm{S}}^{*}(\mathrm{~S}), \omega_{\mathrm{i}}^{*}, \omega_{-\mathrm{i}}^{*}(\mathrm{~S})\right) \geq \pi_{\mathrm{i}}\left(\omega_{\mathrm{S}}^{*}(\mathrm{~S}), \omega_{\mathrm{i}}(\mathrm{S}), \omega_{-\mathrm{i}}^{*}(\mathrm{~S})\right) \quad \forall \omega_{\mathrm{i}}(\mathrm{S}) .
\end{aligned}
$$

where $\omega_{\mathrm{S}}(\mathrm{S})$ is the economic strategy vector of coalitions $\mathrm{S}_{1} \ldots \mathrm{S}_{\mathrm{k}}, \omega_{-\mathrm{S}}(\mathrm{S})$ the vector of all other countries not belonging to $S_{1} \ldots S_{k}, \omega_{i}(S)$ the strategy of non-signatory $i$, and $\omega_{-i}(S)$ the strategy vector of all other non-signatories except i under coalition structure S. An asterisk denotes equilibrium strategies.

Computationally, this implies that non-signatories will choose their economic strategies so as to maximize their individual payoff $\pi_{\mathrm{i}}(\omega)$, whereas all signatories $\mathrm{j} \in \mathrm{S}_{\mathrm{h}}$ jointly maximize the aggregate payoff of their coalition $\mathrm{S}_{\mathrm{h}}$. Strategically, this means that the behaviour of non-signatories towards all other countries is selfish and non-cooperative; signatories behave cooperatively towards their fellow members (otherwise cooperation would not be worthwhile analyzing), but non-cooperatively towards outsiders. Economically, this means strategies are group (but not globally) efficient within coalition S.

\footnotetext{
₹ This simple theoretical framework has often been adopted in the literature on international environmental agreements where the assumption of a coalition structure with a single coalition is the most obvious and realistic and where the game is characterized by positive externalities. A more general framework is sometimes used in coalition theory (Bloch, 2003) but would not be useful for the purpose of this paper.

${ }^{\S}$ This has been called a partial agreement Nash equilibrium by Chander and Tulkens (1997). Our analysis is in line with the mainstream of the literature on coalition theory. For an overview, see Bloch (2003) and Yi (2003).
} 
Given that the second stage of the game has been solved, let us define $v_{i}(S)=\pi_{i}\left(\omega^{*}(S)\right)$ as the valuation of country $\mathrm{i}$ if the coalition structure $\mathrm{S}$ forms. This definition succinctly summarizes all information relevant to the second stage.

For the first stage, a Nash equilibrium in terms of participation can be computed. ${ }^{* *}$ The following two conditions must be met:

Profitability. A multiple coalition structure $S$ is profitable if, when a coalition $S_{i} \in S$ forms, all players $\mathrm{j} \in \mathrm{S}_{\mathrm{i}}, \forall \mathrm{i}$, receive a payoff larger than when no coalition forms, i.e. $\mathrm{v}_{\mathrm{j}}\left(\mathrm{S}_{\mathrm{i}} ; \mathrm{S}\right) \geq \mathrm{v}_{\mathrm{j}}\left(1 ; \mathrm{S}^{1}\right)$ where $\mathrm{S}^{1}$ is the coalition structure where all players are singleton.

Stability. A multiple coalition structure $\mathrm{S}$ is stable if each coalition $\mathrm{S}_{\mathrm{i}} \in \mathrm{S}$ is internally stable, externally stable and intracoalition stable. It is internally stable if no cooperating player would be better off by leaving the coalition to form a singleton. Formally: $v_{i}\left(S_{i} ; S\right) \geq v_{i}\left(1 ; S^{\prime}\right)$ for all players in the coalition $S_{i}$ and all coalitions in $\mathrm{S}$, where $\mathrm{S}^{\prime}=\mathrm{S} \backslash\left\{\mathrm{S}_{\mathrm{i}}\right\} \cup\left\{\mathrm{S}_{\mathrm{i}}-1,1\right\}$. It is externally stable if no singleton would be better off by joining any coalition belonging to the coalition structure $S$. Formally: $v_{i}(1 ; S) \geq v_{i}\left(S_{i} ; S^{\prime}\right)$ for all players who do not belong to $S_{i}$ or to any other non-trivial coalition in $S$, where $S$ "= $S \backslash\left\{S_{i}, 1\right\} \cup\left\{S_{i}+1\right\}$. It is intracoalition stable if no player belonging to $S_{i}$ would be better off by leaving $S_{i}$ to join any other coalition $S_{j} \in S$. Formally: $v_{i}\left(S_{i} ; S\right) \geq v_{i}\left(S_{j}+1 ; S^{\circ}\right)$ for all players in the coalition $S_{i}$ and all coalitions in $\mathrm{S}$, where $\mathrm{S}^{\circ}=\mathrm{S} \backslash\left\{\mathrm{S}_{\mathrm{i}}, \mathrm{S}_{\mathrm{j}}\right\} \cup\left\{\mathrm{S}_{\mathrm{i}}-1, \mathrm{~S}_{\mathrm{j}}+1\right\}$.

That is, at the equilibrium, no signatory belonging to coalition $S_{i} \in S, \forall i$, has an incentive to leave its coalition in order to become a non-signatory, given the participation decisions of all other countries. By the same token, no non-signatory has an incentive to join coalition $\mathrm{S}_{\mathrm{i}}$, given the decisions of all other countries. And no player wants to move from one coalition to another one.

As shown below, the set of stable coalitions may be empty, i.e. the asymmetries between players and/or their different incentives to free-ride and/or to join a specific coalition may be such that no coalition structure is both profitable and stable.

\subsection{The Empirical Model}

The analysis of the possible outcomes of the dynamic process that defines the incentives to participate in a climate agreement has been carried out by using a modified version of Nordhaus' RICE model (Cf. Nordhaus and Yang, 1996) in which endogenous and induced technical change are modelled. In

\footnotetext{
** This definition of coalitional stability is due to d'Aspremont et al. (1983) and has been frequently applied in the literature on international environmental agreements, as for instance by Barrett (1994), Carraro and Siniscalco (1993), Hoel (1992) and by many scholars afterwards.
} 
our version of the model, called FEEM-RICE (Cf. Buonanno et al., 2002), technical change performs a twofold role: on the one hand, via increasing returns to scale, it yields endogenous growth; on the other hand, by affecting the emission/output ratio, it accounts for the adoption of cleaner and energysaving technologies. ${ }^{\dagger \dagger}$

In the model, six countries/regions (US, EU, Japan (JPN), former Soviet Union (FSU), China (CHN) and the rest of the world (ROW)) optimally set the intertemporal values of four strategic variables: investments, $R \& D$ expenditure, abatement effort and net demand for emission permits. Countries play the two-stage game described in sub section 3.1. Given the interdependency of countries' decisions, and the dynamic nature of the RICE model, the equilibrium value of the control variables is the solution of a dynamic Nash game. More precisely, we adopt the PANE - equilibrium concept introduced by Eyckmans and Tulkens (2002) and used in several other papers (Cf. Eyckmans and Finus, 2005; Carraro, Eyckmans and Finus, 2006).

We can provide here only a short overview of the FEEM-RICE model, a more detailed description can be found in the Appendix (see also Eyckmans and Tulkens, 2002 for a description of a modified version of the RICE model). The economic module of FEEM-RICE consists of a long-term dynamic, perfect foresight Ramsey type optimal growth model with endogenous technical change. The decision variables are investments, $R \& D$ expenditure and carbon emission abatement. The carbon cycle and temperature change module are the same as in RICE. In each world region and at every time period t, a regional budget equation describes how gross production, $\mathrm{Y}_{\mathrm{i}, \mathrm{t}}$, can be allocated to consumption, $\mathrm{Z}_{\mathrm{i}, \mathrm{t}}$, investment, $\mathrm{I}_{\mathrm{i}, \mathrm{t}}$, emission abatement costs, $\mathrm{Y}_{\mathrm{i}, \mathrm{t}} \mathrm{C}_{\mathrm{i}}\left(\mu_{\mathrm{i}, \mathrm{t}}\right)$, and climate change damages, $\mathrm{Y}_{\mathrm{i}, \mathrm{t}} \mathrm{D}_{\mathrm{i}}\left(\Delta \mathrm{T}_{\mathrm{t}}\right)$.

Gross production can be interpreted as "potential GDP", that is, what could be produced in the absence of the climate change problem. Abatement costs are an increasing and convex function of emission abatement effort. Abatement effort measures the relative emission reduction compared to the Business-as-usual scenario (BAU) without any abatement policy. Climate change damages are an increasing and convex function of temperature change $\Delta \mathrm{T}_{\mathrm{t}}$. Abatement costs and climate change damages are treated as proportions of "potential production". Hence, total costs and damages are the product of costs and damages with "potential” production $\mathrm{Y}_{\mathrm{i}, \mathrm{t}}$, respectively.

Every region is characterised by a production function that maps combinations of capital stock $\mathrm{K}_{\mathrm{i}, \mathrm{t}}$ and labour input $\mathrm{L}_{\mathrm{i}, \mathrm{t}}$ into output. The production technology is assumed to satisfy constant returns to scale of the Cobb-Douglas type. Labour supply is assumed to be inelastic. Capital accumulation is described

\footnotetext{
${ }^{\dagger \dagger}$ The FEEM-RICE model has already been used in Bosello et al. (2003), Bosetti et al. (2005), Buchner and Carraro (2005a, 2005b, 2006), Buchner et al. (2005) and Buonanno et al. (2002).
} 
in the standard way. In our version of the RICE model, investment is divided into physical and R\&D investments. The former adds up to the existing stock of capital. The second modifies the productivity parameter in the production function and also affects the emission-output ratio (see the Appendix for a detailed presentation of the equations).

Production gives rise to emissions of greenhouse gases. In FEEM-RICE, emissions are proportional to "potential" output. Emissions accumulate in the atmosphere according to a standard linear stock externality accumulation process. Carbon concentration is translated into temperature change according to an increasing function. Welfare of each country is measured by its aggregate lifetime discounted consumption.

In addition to the model structure, two assumptions qualify our results ${ }^{\ddagger \ddagger}$. First, all countries/regions which adhere to the Kyoto/Bonn agreement are assumed to meet their Kyoto target from 2010 onward. ${ }^{\S \S}$ We therefore adopt the so-called “Kyoto forever” hypothesis (Manne and Richels, 1999). Our reference to the Kyoto/Bonn agreement is partly imprecise since, for the sake of brevity, we will at times call the "Kyoto protocol” or "Kyoto/Bonn agreement” a "Kyoto forever" scenario.

Second, cooperating countries are assumed to adopt cost-effective environmental policies. In particular, cost-effective market mechanisms (e.g. emission trading) are chosen over "command-andcontrol" measures in order to guarantee an efficient implementation of the environmental targets adopted within the coalition. Please note that Annex B countries that belong to a coalition and therefore engage in emissions trading face their Kyoto targets, whereas China is assumed to agree to a $10 \%$ reduction of emissions with respect to the BAU scenario over the whole time horizon if it accepts to participate in a coalition (and in emissions trading). If various sub-global coalitions form, then they are assumed to behave independently, without a link between them (i.e., there is no trade between all regional blocs on a common market). This latter assumption mimics the present configuration of permit markets (Cf. Victor, 2006).

\footnotetext{
\# Please note also that our analysis focuses only on CO2. There are other man-made greenhouse gases and the Kyoto Protocol takes some of them into account. Moreover, both the Bonn agreement and the subsequent Marrakech deal emphasise the role of sinks in meeting the Kyoto targets. As shown by several recent analyses (e.g. Manne and Richels, 2001; Jensen and Thelle, 2001), the inclusion of the other greenhouse gases and of sinks would further reduce mitigation costs .

$\S \S$ The use of the "Kyoto forever" hypothesis may be seen as a strong assumption. However, the CO2 concentration levels implicit in this assumption (if FEEM-RICE is a good description of the world) coincide with those in the A1B scenario (IPCC, 2001) which can be considered the "median" scenario among those currently proposed. We thus use the "Kyoto-forever" hypothesis not because it represents a realistic scenario, but as a benchmark with respect to which policy alternatives can be compared.
} 
Using the FEEM-RICE model, we will analyse the incentives to move away from the present situation where the EU, Japan and Russia are committed to complying with their Kyoto targets and where the other countries/regions are free to determine their climate policy unilaterally. Therefore, our benchmark case, or business-as-usual scenario, to which we compare different potential climate regimes, is the coalition formed by the Annex $B_{-u s}$ countries.

Our focus is on post-2012 scenarios. We assume that a global agreement is only one of the possible outcomes of climate negotiations. Countries are also free to form regional or sub-global agreements. Therefore, we consider situations in which countries that now belong to the Kyoto coalition may decide, according to their own economic interests, to leave the Kyoto coalition and cooperate on GHG emission control with other countries/regions ${ }^{* * *}$. The time horizon over which climate policy is optimised is 2010-2100. When analysing the decision to leave or join a coalition, we adopt the "open membership” rule, that implies that non-members can join and leave an existing coalition even without the consensus of the other members.

\section{Results}

In this section, we plan to identify the stable coalition structures of the game. For this purpose, we compare different regimes both with respect to the benchmark climate coalition formed by the Annex $\mathrm{B}_{\text {-us }}$ countries, and with respect to all other different regimes. Comparisons will be performed in terms of each region's payoff (total domestic welfare). In order to limit the number of coalition structures to be compared, we simplify the policy framework as follows. First, given that the inclusion of the leastdeveloped countries is very unlikely in the next stages of climate negotiations, the rest of the world (ROW) is exempted from potential short- to medium- term emission reduction commitments. The player ROW is thus a free-rider in all our policy scenarios. Symmetrically, given its strong commitments to emission reductions, Europe cannot be a free rider, i.e. it always belongs to a climate coalition.

Given these constraints and the FEEM RICE model, our first conclusion is as follows. No coalition structure involving the five negotiating players (ROW is always a free rider) is internally and externally stable. This result is consistent with previous findings using the RICE model (see, for example, Bosello et al. 2003 - where only one coalition structure was found to be stable - and

\footnotetext{
*** Notice that the rest of the world (ROW) has been exempted from possible future climate commitments due to policy indications, which suggest that an inclusion of these countries is very unlikely in the next stage of climate negotiations.
} 
Carraro, Eyckmans and Finus, 2006 - where no stable coalition could be found in the absence of transfers). ${ }^{\dagger \dagger}$

This result suggests that stability analysis does not seem to be useful to analyse the future prospects of climate policy unless one wants to conclude that only unilateral measures are likely to be implemented in the future. This obviously depends on the model used for the empirical analysis and on the abatement targets assumed for the next negotiation stages (in Buchner and Carraro, 2005b, we analysed whether different future emission targets could increase the prospect for a stable coalition, but this was not the case). It also depends on the absence of transfers (in Carraro, Eyckmans and Finus, 2006, the introduction of transfers is shown to yield some stable coalition structures) and on the initial allocation of emission rights (Bosello et al., 2003, show that more equitable allocations slightly increase the number of stable coalition structures).

In this paper we adopt a different approach. Rather than looking for additional instruments (transfers, allocations of emission rights, linkages with other economic issues ${ }^{\ddagger \ddagger \ddagger}$ ) that may yield a stable coalition structure, we will use the payoffs associated to all feasible coalition structures as indicators of each region/country's preferences for different alternative coalition structures, i.e. we will identify the most preferred coalition structures for each country/region of the world and then analyse whether these preferences can help identifying a coalition which is likely to be chosen by an ample set of countries. In doing so, we will also check whether a given coalition structure is at least profitable to all countries/regions belonging to a coalition.

Our results are summarised by Tables $1 \mathrm{a}, 1 \mathrm{~b}$ and 2 . Tables $1 \mathrm{a}, 1 \mathrm{~b}$ show the ranking of climate coalition structures according to domestic welfare. Table 2 shows the ranking of climate regimes according to global welfare and global emissions. These tables may provide information that enables us to identify the most likely behaviour of countries in future climate negotiations (at least to the extent that economic incentives affect climate negotiations).

Let us first focus on the US. The two most preferred coalition structures are the ones in which the US is not involved in any climate coalition (see Table 1a). Note that the US's most preferred regime is the present Annex B-us coalition. However, the US's most preferred regime when it participates in a climate coalition is the one in which the US cooperates (and trades) with China, whilst a second

\footnotetext{
${ }^{\mathrm{H+ \dagger}}$ The result in this paper is slightly stronger than in Bosello et al. (2003) because of the additional constraints imposed on some players of the game (e.g. ROW is always is a free rider) and because of the more demanding target assumed for China.

非 The role of issue linkage in explored in Buchner et al. (2005).
} 
cooperative bloc is formed by the Annex $\mathrm{B}_{\text {-us }}$ coalition, i.e. the US prefers the coalition structure [(JPN, EU, FSU), (USA, CHN)].

The ranking of climate coalition structures for the other two main industrialised countries - EU and Japan - shows some similarities. Both the EU and Japan rank the present Kyoto coalition very low, thus suggesting that a post-2012 change is likely. And both rank cooperation with China quite high. Indeed, the coalition (JPN, EU, CHN, FSU) is the EU's most preferred regime, while Japan ranks first the coalition structure consisting of two blocs, the Asian bloc (JPN and CHN) on the one hand and the European bloc (EU plus FSU) on the other. In both cases, the EU and Japan can profit from either a large emission permit market or at least the presence of an important permit supplier, which implies a low permit price and thus low abatement costs. The worst regime for the EU (and for Japan) is the one in which the EU and Japan form a coalition without having any large permit supplier at their disposal.

Table 1a. The ranking of climate regimes according to domestic total welfare: US, JPN and EU

\begin{tabular}{|c|c|c|}
\hline USA & JPN & EU \\
\hline (JPN, EU, FSU) & (EU, FSU) & (JPN, EU, CHN, FSU) \\
\hline (JPN, CHN) \& (EU, FSU) & (JPN, CHN) \& (EU, FSU) & (USA, JPN, EU, CHN, FSU) \\
\hline (JPN, EU) & (JPN, EU, CHN, FSU) & (JPN,CHN) \& (EU, FSU) \\
\hline (EU, FSU) & (USA, JPN, EU, CHN, FSU) & (EU, FSU) \\
\hline (JPN, EU, CHN, FSU) & (JPN, EU, FSU) \& (USA, CHN) & (JPN, EU, FSU) \& (USA, CHN) \\
\hline (JPN, EU, FSU) \& (USA, CHN) & (JPN, EU, FSU) & (JPN, EU, FSU) \\
\hline (USA, JPN, EU, CHN, FSU) & (USA, JPN, EU, FSU) & (USA, JPN, EU, FSU) \\
\hline (JPN, EU) \& (USA, FSU) & (JPN, EU) \& (USA, FSU) & (JPN, EU) \& (USA, FSU) \\
\hline (USA, JPN, EU, FSU) & (JPN, EU) & (JPN, EU) \\
\hline
\end{tabular}

Note that the coalition structure [(JPN, EU, FSU), (USA, CHN)], i.e. the climate regime in which the US and China cooperate within one bloc, whilst EU, FSU and Japan cooperate within a second bloc, is ranked fifth both by the EU and by Japan. However, there are also some differences in the preferences of the EU and Japan. In particular, large coalitions are more preferred by the EU than by Japan.

Let us analyse the preferences of less developed countries - China and the FSU. China acts as a rational free-rider. Its preferred regime is the two-bloc regime in which the EU cooperates with Japan and the US with Russia, and its second-best option is also a regime in which China free-rides. China's 
most preferred regime when it participates in a climate coalition is the one in which China cooperates (and trades) with the US, whilst a second cooperative bloc is formed by the Annex $\mathrm{B}_{\text {-us }}$ coalition. The possibility of an Asian bloc appears to restrict its potential advantage with respect to gains from the emission market.

Finally, the FSU is penalised by China's participation in a climate regime because China has lower marginal abatement costs and therefore replaces Russia as the large supplier of emission permits. Therefore, the FSU would like to avoid coalitions in which China also participates. The FSU's most preferred regime is the Annex $\mathrm{B}_{\text {-us }}$ coalition, where China is not involved and Russia therefore represents the only permit seller.

Table 1b. The ranking of climate regimes according to domestic total welfare: $\mathrm{CHN}$ and FSU

\begin{tabular}{|c|c|}
\hline CHN & FSU \\
\hline (JPN, EU) \& (USA, FSU) & (JPN, EU) \\
\hline (USA, JPN, EU, FSU) & (USA, JPN, EU, FSU) \\
\hline (JPN, EU, FSU) & (JPN, EU, FSU) \\
\hline (JPN, EU) & (JPN, EU, FSU) \& (USA, CHN) \\
\hline (EU, FSU) & (JPN, EU, CHN, FSU) \\
\hline (JPN, EU, FSU) \& (USA, CHN) & (JPN, FHU) \\
\hline (USA, JPN, EU, CHN, FSU) $\&$ (EU, FSU) \\
\hline (JPN, EU, CHN, FSU) & (JPN, EU) \& (USA, FSU) \\
\hline (JPN, CHN) \& (EU, FSU) & (USA, JPN, EU, CHN, FSU) \\
\hline
\end{tabular}

What are the policy lessons that can be derived from Tables 1a and 1b? As seen above, US and China have a strong incentive to free-ride, namely to set their environmental policy unilaterally, thus profiting from the abatement levels set for the Kyoto coalition countries. In particular, the Annex $\mathrm{B}$-us coalition is the US's most preferred regime and has been ranked third by China. The Annex B-Us coalition is also good for the FSU, for which this is the second-best outcome when it decides not to free-ride. 
EU and Japan have a strong incentive to maintain cooperation with a large permit seller, e.g. at least with the FSU. Indeed, the worst coalition structures for the EU and for Japan are the ones in which the EU and Japan form a coalition without either China or the FSU.

In short, the climate coalition structure where only the EU, Japan and the FSU cooperate is fairly robust in terms of economic incentives (even though not stable), but highly ineffective from an environmental viewpoint, as is demonstrated by Table 2. Paradoxically, this regime is not very welcome by the EU and Japan, but is among the most preferred ones by the other countries.

What could be an alternative climate regime with some economic incentives for the participating countries/regions? It is clear that Russia does not like to cooperate with China, because of the losses that it would suffer in the permit market. China would like to free ride, but if it cooperates, it prefers the coalition structure [(JPN, EU, FSU), (USA, CHN)]. Likewise for the US. The EU would prefer a large coalition, whereas Japan likes a regional two-bloc coalition (when it does not free ride). Therefore, if the US and China would decide to cooperate to control their GHG emissions, they may sign a bilateral agreement rather than joining a large global coalition. The conclusion is that the coalition structure [(JPN, EU, FSU), (USA, CHN)] has some likelihood to replace the existing coalition structure [(JPN, EU, FSU), USA, CHN], even though the latter is fairly robust.

Let us look at the coalition structure [(JPN, EU, FSU), (USA, CHN)] in more detail. Is it profitable to all countries? Is it environmentally effective? Are there elements in the actual policy process that suggest that this coalition structure may not be unrealistic? Let us first look at this latter aspect. China's decision to ratify the Kyoto Protocol demonstrates that the country is aware that benefits from ratification could be high because China is the largest permit seller. Chinese officials emphasise that the government will voluntarily try to restrict the growth of $\mathrm{CO} 2$ emissions, but is strictly opposing binding GHG reduction targets (The Japan Times, Jan. $26^{\text {th }}$, 2002), notwithstanding recent signals that the country might soften its position in the future. Overall, the Chinese strategy appears to be strongly linked to the moves of the US, and together these two countries could accomplish a break-through in international climate cooperation.

Without binding commitments or with very mild abatement targets and given the consequent high amount of permits which can be supplied, China is a very attractive partner in climate change control activities. This is why the US could convince China to cooperate under a joint climate pact. In this way, the US could achieve two goals: (i) satisfy domestic political requirements by involving developing countries in their climate strategy; (ii) reap high benefits from a large joint emissions market (the US and China together account for more than one-third of the world-wide CO2 emissions and this share is becoming larger and larger). In particular, the US could drastically decrease its 
abatement costs through emission trading, and China could profit from selling a large amount of permits.

What are the main economic and environmental implications of this coalition structure? Figure 1 below shows that both the US and CHN lose with respect to the case in which they free-ride. Therefore the coalition structure is not profitable. However, the loss for the US is small and could be largely compensated by some ancillary benefits from GHG emission abatement that are not taken into account in our model.

Figure 1: A third climate regime with two blocs: 1) US and CHN; 2) EU, FSU and Japan

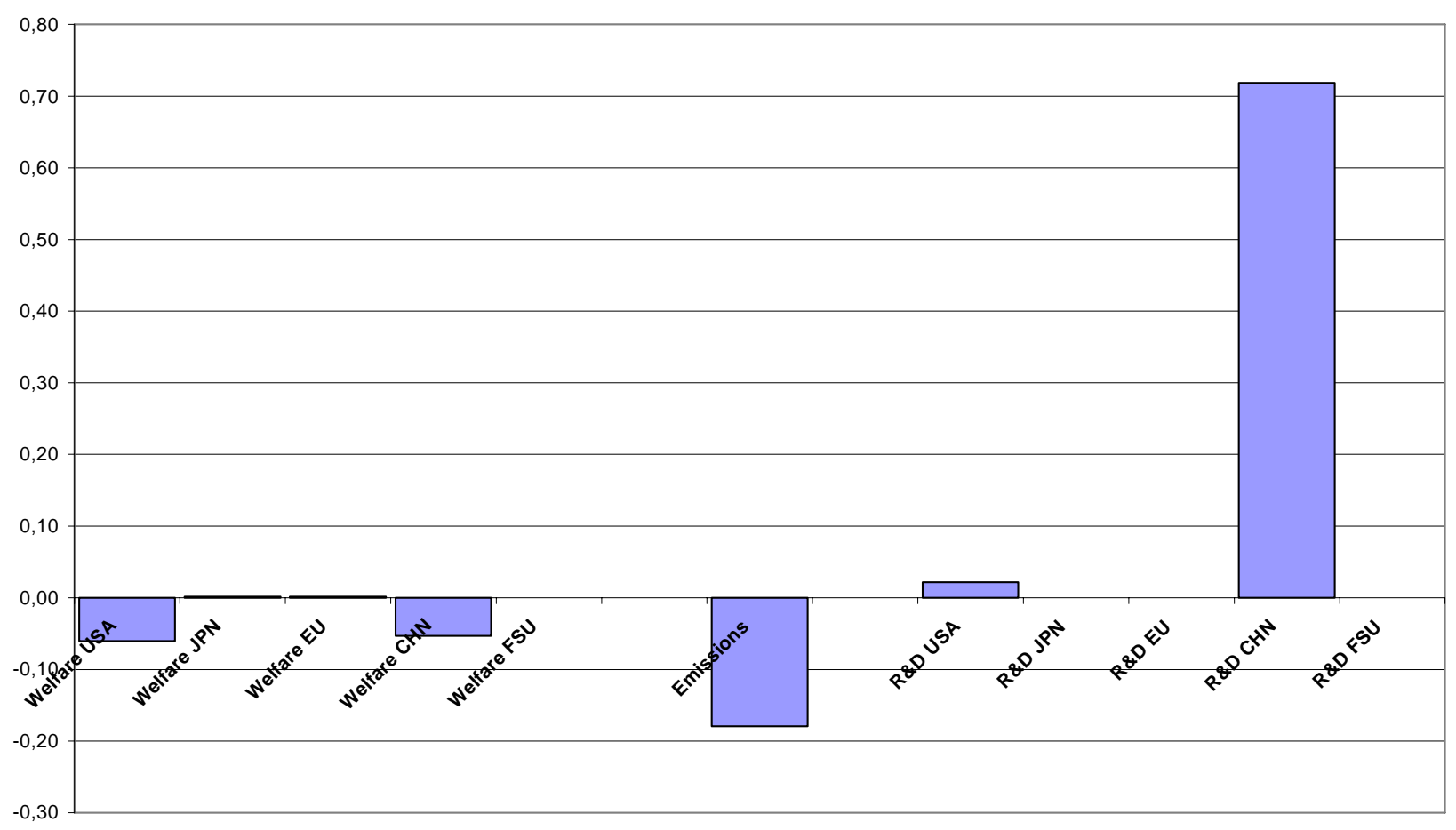

The loss for China is also small and ancillary benefits, both on the environmental and economic side, could be large. Note that, as discussed above, this coalitions structure is the most preferred one by both the US and China when they do not free-ride. Namely, it is the most preferred one among those in which they undertake some cooperative emission abatement.

The coalitions structure [(JPN, EU, FSU), (USA, CHN)] is slightly beneficial for the Kyoto climate bloc consisting of the European Union, Japan and the FSU, because of the enhanced environmental effectiveness of this two-bloc regime. Indeed, GHG emissions are almost $20 \%$ lower than in the 
benchmark case (see Figure 1). This two-bloc climate regime is also characterised by a large expansion of China's R\&D investments. China over-invests in $R \& D$ to increase its sales in the bilateral emission trading market. The segmentation of the trading market explains why $R \& D$ investments within the benchmark Annex $\mathrm{B}_{\text {-us }}$ coalition do not change. However, if the comparison is made with the coalition (Annex $\mathrm{B}_{\text {-us }}+$ China), then it can be seen that $R \& D$ investments in this climate regime are higher for all Annex $\mathrm{B}_{\text {-us }}$ countries. The reason is again the larger marginal abatement costs when China is not a seller in the permit market. This induces higher investments in $R \& D$ in the EU and Japan and also strategic R\&D investments in the FSU, which will find it optimal to increase its supply of permits.

Summing up, the coalition structure [(JPN, EU, FSU), (USA, CHN)] is neither stable nor profitable according to the definitions of Section 2 of this paper. However, it is profitable and stable for the coalition (JPN, EU, FSU). China and the US suffer a small loss when cooperating inside this coalition structure, but the loss is the smallest one among all possible coalition structures in which the US and China cooperate.

The above conclusions are based on a decentralised analysis of each country's incentives to join a climate coalition. However, it would be important to assess what a central planner would do when his/her goal is the maximisation of global welfare. The answer is provided by Table 2, second column, which shows that global welfare - which includes welfare of free-riders - is maximised when the coalition structure [(JPN, EU, CHN, FSU), USA] forms ${ }^{\S \S}$. Second is the coalition structure [(JPN, $\mathrm{CHN}),(\mathrm{EU}, \mathrm{FSU}), \mathrm{USA}]$, whereas the climate regime where the US cooperates with China and the Annex $B_{\text {-us }}$ forms as a second bloc, i.e. [(JPN, EU, FSU), (USA, CHN)], is only sixth. Notice, that global welfare seems to be maximised when the US does not belong to a climate coalition, which suggests that an unconstrained growth of the US economy may be beneficial to the world economy, despite the damage to the environment.

Table 2. The ranking of climate regimes according to global welfare and global GHG emissions

\begin{tabular}{|c|c|}
\hline Global GHG Emissions & Global Welfare \\
\hline (USA, JPN, EU, CHN, FSU) & (JPN, EU, CHN, FSU) \\
\hline (JPN, EU, FSU) \& (USA, CHN) & (JPN, CHN) \& (EU, FSU) \\
\hline (JPN, EU) \& (USA, FSU) & (EU, FSU) \\
\hline (USA, JPN, EU, FSU) & (JPN, EU, FSU) \\
\hline
\end{tabular}

\footnotetext{
$\S \S \S$ The fact that the grand coalition does not appear first in the ranking of global welfare is due to the exemption of ROW from future emission abatement commitments, i.e. in our analysis ROW is always a free rider.
} 


\begin{tabular}{|c|c|}
\hline (JPN, CHN) \& (EU, FSU) & (USA, JPN, EU, CHN, FSU) \\
\hline (JPN, EU, FSU) & (JPN, EU, FSU) \& (USA, CHN) \\
\hline (JPN, EU, CHN, FSU) & (JPN, EU) \\
\hline (EU, FSU) & (USA, JPN, EU, FSU) \\
\hline (JPN, EU) & (JPN, EU) \& (USA, FSU) \\
\hline
\end{tabular}

Also notice that the two-bloc climate regime [(JPN, EU, FSU), (USA, CHN)] would provide the second largest possible benefit for the environment (see Figure 2, first column).

Summarising, if for some environmental, economic or political reasons, the US and China decide to cooperate to control their GHG emissions, the probability to sign a bilateral agreement rather than joining a large global coalition is quite high. This situation, which corresponds to the coalition structure [(JPN, EU, FSU), (USA, CHN)], slightly increases welfare in the EU, Japan and the FSU, at least with respect to the present Kyoto coalition (see Figure 1). Therefore, the Annex $B_{\text {-us }}$ countries may accept a two bloc regime, where the US and CHN cooperate on emission abatement and trade permits in a bilateral permit market. The economic loss for the US and CHN would be small with respect to the situation in which they free-ride, but the global environmental benefits would be large.

\section{Conclusions}

The conclusions emerging from the above analysis can be summarised as follows. A move from the present climate regime in which the US and China do not cooperate to reduce their GHG emissions is not likely, at least in the short-run. The US is more likely to adopt unilateral policies than to join a coalition to control GHG emissions. However, it is unlikely that, at least in the medium term, the US continues to reject any form of cooperation on climate change control. If the US decides to cooperate, the climate regime which is least opposed (in terms of net economic benefits) by the negotiating countries is the one in which China and the US cooperate bilaterally and the Annex $\mathrm{B}_{\text {-us }}$ countries form a parallel coalition.

Of course, the above findings must be taken cautiously. First, because they are based only on the analysis of economic incentives, whereas political decisions could be taken on the basis of other types of incentives. Second, because we did not account for the link between climate negotiations and other international negotiation processes (e.g. on crime, trade, terrorism, technology, etc.). Third, because the FEEM-RICE model used in this study is a simplified representation of the world economic system, even though it captures the main economic mechanisms and the related incentive schemes. Additional research with other models would be beneficial. 
Nonetheless, the results derived from the empirical analysis proposed in this paper are quite consistent with suggestions and results proposed in the game-theory literature (where countries' asymmetries are usually neglected). This suggests that the analysis in this paper is likely to capture the relevant economic incentives and may therefore serve to provide indications on the prospective future evolution of negotiations on climate change control. 


\section{References}

Aumann, R. and J. Drèze (1974), Cooperative Games with Coalition Structures, International Journal of Game Theory, 3, 217-37.

Barrett, S. (1994) Self-Enforcing International Environmental Agreements, Oxford Economic Papers, 46, 878-894.

Barrett, S. (2002), Environment and Statecraft. Oxford: Oxford University Press.

Bloch, F. (1995) Endogenous Structures of Associations in Oligopolies, RAND Journal of Economics, 26, 537-556.

Bloch, F. (1996) Sequential Formation of Coalitions in Games with Externalities and Fixed Payoff Division, Games and Economic Behavior, 14, 90-123.

Bloch, F. (2003), Noncooperative Models of Coalition Formation in Games with Spillovers, in C. Carraro (ed.), The Endogenous Formation of Economic Coalitions, E.Elgar, Cheltenham.

Bosello, F., Buchner, B. and C. Carraro (2003), Equity, Development and Climate Change Control, Journal of the European Economic Association, 1 (2-3), 601-611.

Bosetti, V., C. Carraro and M. Galeotti (2005), The Dynamics of Carbon and Energy Intensity in a Model of Endogenous Technical Change, FEEM Working Paper 6.2005; forthcoming in the Energy Journal.

Buchner, B. and C. Carraro (2005a), Economic and Environmental Effectiveness of a Technologybased Climate Regime, Climate Policy, 4, 229-248.

Buchner, B. and C. Carraro (2005b), Modelling Climate Policy. Perspectives on Future Negotiations, Journal of Policy Modeling, 27 (6), 711-732.

Buchner, B. and C. Carraro (2006), US, China and the Economics of Climate Negotiations, International Environmental Agreements. Law Politics Economics.

Buchner, B., C. Carraro, I. Cersosimo and C. Marchiori (2005), Back to Kyoto? US Participation and the Linkage between R\&D and Climate Cooperation. In A. Haurie and L. Viguier, eds., Coupling Climate and Economic Dynamics, Kluwer Academic Publishers, Dordrecht, 173-204.

Buonanno, P., C. Carraro, and M. Galeotti (2002), Endogenous Induced Technical Change and the Costs of Kyoto, Resource and Energy Economics, 524, 11-35.

Carraro, C. (1998), Beyond Kyoto: A Game Theoretic Perspective, in the Proceedings of the OECD Workshop on "Climate Change and Economic Modelling. Background Analysis for the Kyoto Protocol”, Paris, 17-18.9, 1998.

Carraro, C. (1999), The Structure of International Agreements on Climate Change, in C. Carraro, ed., International Environmental Agreements on Climate Change, Kluwer Academic Pub.: Dordrecht.

Carraro, C., ed. (2003), The Endogenous Formation of Economic Coalitions, E.Elgar, Cheltenham.

Carraro, C., Eyckmans, J. and M. Finus (2006), Optimal Transfers and Participation Decisions in International Environmental Agreements, forthcoming in the Review of International Organizations.

Carraro, C. and C. Marchiori (2003), Stable Coalitions, in C. Carraro, ed., The Endogenous Formation of Economic Coalitions, E.Elgar, Cheltenham.

Carraro, C. and D. Siniscalco (1993), Strategies for the International Protection of the Environment, Journal of Public Economics, 52, 309-328.

Chander, P. and H. Tulkens (1997), The Core of an Economy with Multilateral Environmental Externalities, International Journal of Game Theory, 26, 379-401. 
Coe, D.T. and E. Helpman (1995), International R\&D Spillovers, European Economic Review, 39, 859-887.

D’Aspremont, C.A., A. Jacquemin, J.J. Gabszewicz and J. Weymark. 1983. On the Stability of Collusive Price Leadership, Canadian Journal of Economics, 16, 17-25.

Demange, G., Ray, D., and M. Wooders, eds. (2005), Group Formation in Economics. Networks, Clubs and Coalitions, Cambridge University Press, Cambridge.

Egenhofer, C., W. Hager and T. Legge (2001), Defining Europe's Near Abroad in Climate Change: A Russian-EU Alliance - Sub-global Bargaining to Further International Environmental Agreements. CEPS Discussion Paper.

Eyckmans, J. and H. Tulkens (2002), Simulating Coalitionally Stable Burden Sharing Agreements for the Climate Change Problem, CORE Discussion Paper 9926 and CLIMNEG Working Paper 18, Université Catholique de Louvain, Belgium.

Eyckmans, J. and M. Finus (2006), Measures to Enhance the Success of Global Climate Treaties, this volume.

Finus, M. and B. Rundshagen (2003), Endogenous Coalition Formation in Global Pollution, in C. Carraro, ed., The Endogenous Formation of Economic Coalitions, E.Elgar, Cheltenham.

Goulder, L.H. and K. Mathai (2000), Optimal CO2 Abatement in the Presence of Induced Technological Change, Journal of Environmental Economics and Management, 39, 1-38.

Griliches, Z. (1979), Issues in Assessing the Contribution of R\&D to Productivity Growth, Bell Journal of Economics, 10, 92-116.

Griliches, Z. (1984), R\&D, Patents, and Productivity, Chicago: University of Chicago Press.

Hoel, M. 1992. International Environmental Conventions: The Case of Uniform Reductions of Emissions, Environment and Resource Economics, 2, 141-159.

IPCC (2001), Third Assessment Report, Cambridge University Press, Cambridge.

Jensen, J. and M.H. Thelle (2001), What are the gains from a multi-gas strategy? FEEM Working Paper 84.01, Milan.

Manne, A. and R. Richels (1999), The Kyoto Protocol: a cost-effective strategy for meeting environmental objectives? in J. Weyant, ed., The Cost of the Kyoto Protocol: A Multi-Model Evaluation, Special Issue of the Energy Journal.

Manne, A.S. and R.G. Richels (2001), US Rejection of the Kyoto Protocol: The Impact on Compliance Costs and CO2 Emissions. Working Paper 01-12, AEI-Brookings Joint Center for Regulatory Studies.

Nordhaus, W.D. and Z. Yang (1996), A regional dynamic general-equilibrium model of alternative climate-change strategies, American Economic Review, 4, 741-765.

Nordhaus, W.D. (1999), Modelling Induced Innovation in Climate-Change Policy, paper presented at the IIASA Workshop on Induced Technological Change and the Environment, Laxenburg, June 21-22.

Ray, D. and R. Vohra (1997), Equilibrium Binding Agreements, Journal of Economic Theory, 73, 3078.

Ray, D. and R. Vohra (1999), A Theory of Endogenous Coalition Structures, Games and Economic Behavior, 26, 286-336.

Reinstein, R. A. (2004), A Possible Way Forward on Climate Change, mimeo, Reinstein \& Associates Inc.

Romer, P. (1990), Endogenous Technological Change, Journal of Political Economy, 94, 1002-1037.

Stewart, R and J. Wiener (2003), Reconstructing Climate Policy, American Enterprise Institute Press. 
The Japan Times, China could help Japan by taking its money and cutting its Kyoto target, January $26^{\text {th }}, 2002$. Online at www.japantimes.com

Victor, D. (2006), "Fragmented Carbon Markets and Reluctant Nations. Implications for the Design of Effective Architectures", paper presented at the international workshop on "Architectures for Agreement: Addressing Global Climate Change in the Post-Kyoto World," Harvard University, May 12-13, 2006.

Yi, S.-S. (1997), Stable Coalition Structures with Externalities, Games and Economic Behaviour, 20, 201-23.

Yi, S.-S. (2003), Endogenous Formation of Economic Coalitions: A Survey of the Partition Function Approach. In: C. Carraro (ed.), Endogenous Formation of Economic Coalitions, Edward Elgar, Cheltenham, UK, pp. 80-127.

Yi, S.-S. and H. Shin (1995), Endogenous Formation of Coalitions: Oligopoly, mimeo, Department of Economics, Dartmouth College.

Weyant, J.P. (1997), Technological Change and Climate Policy Modeling, paper presented at the IIASA Workshop on Induced Technological Change and the Environment, Laxenburg, June 2627.

Weyant, J.P. and T. Olavson (1999), Issues in Modelling Induced Technological Change in Energy, Environmental, and Climate Policy, Environmental Modelling and Assessment, 4, 67-85. 


\section{Appendix: The FEEM-RICE Model}

The FEEM-RICE model is an extension of Nordhaus and Yang's (1996) regional RICE model of integrated assessment, which is one of the most popular and manageable integrated assessment tools for the study of climate change (see, for instance, Eyckmans and Tulkens 2002). It is basically a single sector optimal growth model which has been extended to incorporate the interaction between economic activities and climate. One such model has been developed for each macro region into which the world is divided (USA, Japan, Europe, China, Former Soviet Union, and Rest of the World).

Within each region a central planner chooses the optimal paths of fixed investment and emission abatement that maximise the present value of per capita consumption. Output (net of climate change) is used for investment and consumption and is produced according to constant returns Cobb-Douglas technology, which combines the inputs from capital and labour with the level of technology. Population (taken to be equal to full employment) and technology levels grow over time in an exogenous fashion, whereas capital accumulation is governed by the optimal rate of investment. There is a wedge between output gross and net of climate change effects, the size of which is dependent upon the amount of abatement (rate of emission reduction) as well as the change in global temperature. The model is completed by three equations representing emissions (which are related to output and abatement), carbon cycle (which relates concentrations to emissions), and climate module (which relates the change in temperature relative to 1990 levels to carbon concentrations) respectively.

In our extension of the model, technical change is no longer exogenous. Instead, the issue of endogenous technical change is tackled by following the ideas contained in both Nordhaus (1999) and Goulder and Mathai (2000) and accordingly modifying Nordhaus and Yang's (1996) RICE model. Doing so requires the input of a number of additional parameters, some of which have been estimated using information provided by Coe and Helpman (1995), while the remaining parameters were calibrated so as to reproduce the business-as-usual scenario generated by the RICE model with exogenous technical change.

In particular, the following factors are included: first, endogenous technical change affecting factor productivity is introduced. This is done by adding the stock of knowledge in each production function and by relating the stock of knowledge to R\&D investments. Second, induced technical change is introduced, by allowing the stock of knowledge to affect the emission-output ratio as well. Finally, international technological spillovers are also accounted for in the model.

To determine the optima value of all control variables, including their own GHG abatement strategy, countries play a non-cooperative Nash game in a dynamic setting, which yields an Open Loop Nash 
equilibrium (see Eyckmans and Tulkens, 2002, for an explicit derivation of first order conditions of the optimum problem). This is a situation in which, in each region, the planner maximises social welfare subject to the individual resource and capital constraints and the climate module, given the emission and investment strategies (in the base case) and the R\&D expenditure strategy (in the endogenous technological change case) of all other players.

\section{The Standard Model without Induced Technical Change}

As previously mentioned, it is assumed for the purpose of this model that innovation is brought about by $R \& D$ spending which contributes to the accumulation of the stock of existing knowledge. Following an approach pioneered by Griliches $(1979,1984)$, it is assumed that the stock of knowledge is a factor of production, which therefore enhances the rate of productivity (see also the discussion in Weyant 1997; Weyant and Olavson 1999). In this formulation, R\&D efforts prompt nonenvironmental technical progress, but with different modes and elasticities. More precisely, the RICE production function output is modified as follows:

$$
Q(n, t)=A(n, t) K_{R}(n, t)^{\beta_{n}}\left[L(n, t)^{\gamma} K_{F}(n, t)^{1-\gamma}\right]
$$

where $Q$ is output (gross of climate change effects), $A$ the exogenously given level of technology and $K_{R}, L$, and $K_{F}$ are respectively the inputs from knowledge capital, labour, and physical capital.

In (1), the stock of knowledge has a region-specific output elasticity equal to $\beta_{n}(n=1, \ldots 6)$. It should be noted that, as long as this coefficient is positive, the output production process is characterised by increasing returns to scale, in line with current theories of endogenous growth. This implicitly assumes the existence of cross-sectoral technological spillovers within each country (Romer, 1990). In addition, it should be noted that while allowing for R\&D-driven technological progress, we maintain the possibility that technical improvements can also be determined exogenously (the path of $A$ is the same as that specified in the original RICE model). The stock accumulates in the usual fashion:

$$
K_{R}(n, t+1)=R \& D(n, t)+\left(1-\delta_{R}\right) K_{R}(n, t)
$$

where $R \& D$ is the expenditure in Research and Development and $\delta_{R}$ is the rate of knowledge depreciation. Finally, it is recognised that some resources are absorbed by $R \& D$ spending. That is:

$$
Y(n, t)=C(n, t)+I(n, t)+R \& D(n, t)
$$


where $Y$ is net output (net of climate change effects as specified in the RICE model), $C$ is consumption and $I$ gross fixed capital formation.

At this stage the model maintains the same emissions function as Nordhaus’ RICE model which will be modified in the next section:

$$
E(n, t)=\sigma(n, t)[1-\mu(n, t)] Q(n, t)
$$

where $\sigma$ can be loosely defined as the emissions-output ratio, $E$ stands for emissions and $\mu$ for the rate of abatement effort. The policy variables included in the model are rates of fixed investment and of emission abatement. For the other variables, the model specifies a time path of exogenously given values. Interestingly, this is also the case for technology level $A$ and of the emissions-output ratio $\sigma$. Thus, the model presented so far assumes no induced technical change, i.e. an exogenous environmental technical change, and a formulation of productivity that evolves both exogenously and endogenously. In the model, investment fosters economic growth (thereby driving up emissions) while abatement is the only policy variable used for reducing emissions.

\section{Induced Technical Change}

In the second step of our model formulation, endogenous environmental technical change is accounted for. It is assumed that the stock of knowledge - which in the previous formulation was only a factor of production - also serves the purpose of reducing, ceteris paribus, the level of carbon emissions. Thus, in the second formulation, R\&D efforts prompt both environmental and non-environmental technical progress, although with different modes and elasticities. ${ }^{* * * *}$ More precisely, the RICE emission-output relationship is modified as follows:

$$
E(n, t)=\left[\sigma_{n}+\chi_{n} \exp \left(-\alpha_{n} K_{R}(n, t)\right)\right][1-\mu(n, t)] Q(n, t)
$$

In (4'), knowledge reduces the emissions-output ratio with an elasticity of $\alpha_{n}$, which is also regionspecific; the parameter $\chi_{n}$ is a scaling coefficient, whereas $\sigma_{n}$ is the value to which the emission-output ratio tends asymptotically as the stock of knowledge increases without limit. In this formulation, R\&D contributes to output productivity on the one hand, and affects the emission-output ratio - and therefore the overall level of pollution emissions - on the other .

\footnotetext{
**** Obviously, we could have introduced two different types of R\&D efforts, respectively contributing to the growth of an environmental knowledge stock and a production knowledge stock. Such undertaking however is made difficult by the need to specify variables and calibrate parameters for which there is no immediately available and sound information in the literature.
} 


\section{Knowledge Spillovers}

Previous formulations do not include the effect of potential spillovers produced by knowledge, and therefore ignore the fact that both technologies and organisational structures disseminate internationally. Modern economies are linked by vast and continually expanding flows of trade, investment, people and ideas. The technologies and choices of one region are and will inevitably be affected by developments in other regions.

Following the work of Weyant and Olavson (1999), who suggest that the definition of spillovers in an induced technical change context be kept plain and simple (in the light of a currently incomplete understanding of the problem), disembodied, or knowledge spillovers are modelled (see Romer, 1990). They refer to the R\&D carried out and paid for by one party that produces benefits to other parties which then have better or more inputs than before or can somehow benefit from R\&D carried out elsewhere. Therefore, in order to capture international spillovers of knowledge, the stock of world knowledge is introduced in the third version of the FEEM-RICE model, both in the production function and in the emission-output ratio equation. Equations (1) and (4') are then revised as follows:

$$
Q(n, t)=A(n, t) K_{R}(n, t)^{\beta_{n}} W K_{R}(n, t)^{\varepsilon_{n}}\left[L(n, t)^{\gamma} K_{F}(n, t)^{1-\gamma}\right]
$$

and:

$$
E(n, t)=\left[\sigma_{n}+\chi_{n} \exp \left(-\alpha_{n} K_{R}(n, t)-\theta_{n} W K_{R}(n, t)\right)\right][1-\mu(n, t)] Q(n, t)
$$

where the stock of world knowledge:

$$
W K_{R}(j, t)=\sum_{j \neq i} K_{R}(i, t)
$$

is defined in such a way as not to include a country's own stock.

\section{Emission Trading}

As mentioned above, throughout the analysis we assume the adoption of efficient policies. As a consequence, the model also includes the possibility of emission trading. When running the model in the presence of emission trading, two additional equations are considered: 


$$
Y(n, t)=C(n, t)+I(n, t)+R \& D(n, t)+p(t) N I P(n, t)
$$

which replaces equation (3) and:

$$
E(n, t)=\operatorname{Kyoto}(n)+N I P(n, t)
$$

where $\operatorname{NIP}(n, t)$ is the net demand for permits and Kyoto(n) are the emission targets set in the Kyoto Protocol for the signatory countries and the BAU levels for the non-signatory ones. According to (3'), resources produced by the economy must be devoted, in addition to consumption, investment, and research and development, to net purchases of emission permits. Equation (6) states that a region's emissions may exceed the limit set in Kyoto if permits are bought, and vice versa in the case of sales of permits. Note that $p(t)$ is the price of a unit of tradable emission permits expressed in terms of the numeraire output price. Moreover, there is an additional policy variable to be considered in this case, which is net demand for permits NIP.

In terms of the possibility of emission trading, the sequence whereby a Nash equilibrium is reached can be described as follows. Each region maximises its utility subject to its individual resource and capital constraints, now including the Kyoto constraint, and the climate module for a given emission (i.e. abatement) strategy of all the other players and a given price of permits $p(0)$ (in the first round this is set at an arbitrary level). When all regions have made their optimal choices, the overall net demand for permits is computed at the given price. If the sum of net demands in each period is approximately zero, a Nash equilibrium is obtained; otherwise the price is revised as a function of the market disequilibrium and each region's decision process starts again. 